\title{
Interleukin-6 and C-reactive protein as early markers of sepsis in patients with diabetic ketoacidosis or hyperosmosis
}

\author{
C. A. Gogos ${ }^{1}$, S. Giali ${ }^{1}$, F. Paliogianni ${ }^{2}$, G. Dimitracopoulos ${ }^{2}$, H. P. Bassaris ${ }^{1}$, A. G. Vagenakis ${ }^{1}$ \\ ${ }^{1}$ Department of Medicine, University of Patras, Medical School, Greece \\ ${ }^{2}$ Department of Clinical Microbiology, University of Patras, Medical School, Patras, Greece
}

\section{Abstract}

Aims/hypothesis. An early diagnosis of sepsis in patients with diabetic ketoacidosis and hyperosmolar non-ketotic coma is crucial and could save lives. We studied serum C-reactive protein and interleukin-6 to find out how useful these might be for identifying sepsis.

Methods. Sixty one diabetic patients with ketoacidosis or hyperosmolar non-ketotic coma were enrolled. Patients with signs and symptoms of systemic inflammatory response syndrome were identified. Acutephase reactants, including C-reactive protein and interleukin-6, the main cytokine responsible for the induction of acute-phase proteins, were measured on admission and when patients had clinically improved and were euglycaemic.

Results. A total of 49 out of 61 patients with diabetic ketoacidosis or hyperosmosis had signs of systemic inflammatory response syndrome. Another 27 patients had systemic inflammatory response syndrome and no signs of infection and 22 patients had systemic inflammatory response syndrome due to proven infection. We detected a significant increase in serum C-reactive protein and interleukin- 6 values in patients infected compared with patients not infected with systemic inflammatory response syndrome SIRS. Patients who finally died had much higher levels of these proteins, while there was a prompt reduction of serum C-reactive protein and interleukin-6 early during remission.

Conclusion/interpretation. Diabetic ketoacidosis and hyperosmolar non-ketotic coma can often cause a clinical syndrome resembling systemic inflammatory response syndrome. Determination of serum C-reactive protein and interleukin-6 levels is a useful way of excluding an underlying infection early on as well as confirming and monitoring sepsis. [Diabetologia (2001) 44: 1011-1014

Keywords CRP, IL-6, diabetes mellitus, sepsis, ketoacidosis, hyperosmosis.
Because infections are a major cause of morbidity and mortality in patients with diabetic ketoacidosis (DKA) and hyperosmolar non-ketotic koma (DHNK), the early diagnosis of sepsis and institution

Received: 17 April 2001 and in revised form: 17 April 2001

Corresponding author: C.A.Gogos, M.D., Department of Medicine, University of Patras, Medical School, Rion-Patras 26500, Greece. E-mail: cgogos@med.upatras.gr

Abbreviations: SIRS, systemic inflammatory response syndrome; CRP, C-reactive protein; HPT, haptoglobin; AAT, a-1 antichymotrypsin; TRF, transferrin; OKA, diabetic ketoacidosis; DHNN, diabetic hyperosmotic non-ketotic troma of appropriate antimicrobial treatment in such patients is crucial.

Systemic inflammatory response (SIR) and sepsis reduce the rate of synthesis of selected proteins (i.e., albumin, transferrin, prealbumin, retinol-binding protein and fibronectin), but accelerate the production of acute-phase proteins (CRP, haptoglobin, serum amyloid $\mathrm{A}$, complement $\mathrm{C} 3$, a-1 antithrypsin etc) [1]. Serum C-reactive protein concentrations in healthy individuals have been shown to be uniformly low in the absence of bacterial infections, while values of $4 \mathrm{mg} / \mathrm{dl}$ or more are usually seen in patients with sepsis or, less frequently, with other inflammatory states [2]. The proinflammatory cytokines interleu- 
kin-1 (IL-1), tumour necrosis factor alpha (TNF- $\alpha$ ), and especially Interleukin-6 (IL-6), induce hepatic acute-phase protein synthesis and have been widely studied in patients with SIRS and sepsis [4].

Diabetic ketoacidosis and DHNK are frequently precipitated by infection, accompanied by fever, tachycardia, tachypnoea, leukocytosis and occasionally shock, while the latter are also frequently present in uncomplicated DKA and DHNK stages. In this study, we investigated the effect of diabetic ketoacidosis and hyperosmosis on the inflammatory response and the role of acute-phase proteins and IL-6, as early markers of sepsis in the above situations. We tested the hypothesis that the measurement of IL-6 and CRP early in the course of DKA and DHNK could help to detect underlying infections.

\section{Subjects and methods}

Patient selection. The study included 61 patients with DKA or DHNK and was conducted for a period of 36 months. Informed consent was obtained from all patients or their representatives and the study was approved by the hospital's ethical committee.

A total of $49(80.3 \%)$ patients had signs of SIRS. Of those, $22(44.9 \%)$ proved to have underlying infection based on clinical and/or microbiological criteria (group B), while 27 $(55.1 \%)$ did not (group A). No infection was recorded in any of the 12 non-SIRS patients.

Definition of SIRS/sepsis. Although two of the following criteria would be enough, we used three or more of the criteria to define SIRS, as patients with DKA or DHNK are frequently tachypnoic and tachycardic:

- A temperature of more than $37.8^{\circ} \mathrm{C}$ or less than $36^{\circ} \mathrm{C}$

- A leukocyte count of more than $12 \mathrm{k} / \mu \mathrm{l}$, less than $4 \mathrm{k} / \mu \mathrm{l}$, or more than $10 \%$ immature forms;

- A pulse of more than $90 \mathrm{bpm}$

- Ventilation of more than $20 \mathrm{bpm}$ or $\mathrm{PaCO}_{2}$ of less than $32 \mathrm{~mm} \mathrm{Hg}$

The term "sepsis" was used for patients with SIRS and confirmed infection.

Determination of acute-phase protein concentrations. Serum CRP, $\beta$-2 microglobulin, haptoglobin (HPT), a-1 antichymotrypsin (AAT), transferrin (TRF), immunoglobulins $\operatorname{IgM}, \operatorname{Ig}$ A and $\mathrm{IgG}$ and complement factors $\mathrm{C}_{3}$ and $\mathrm{C}_{4}$ were measured on the patient's admission. Another sample was taken during remission, i.e. when there were no signs of SIRS and glucose concentrations return to normal. Rate nephelometry (Beckman) was used to measure CRP, HPT, AAT, TRF, IgM, IgA, IgG and $\mathrm{C}_{3}, \mathrm{C}_{4}$ We used IMx-ELISA (Abbott Labs, Greece, USA) to measure $\beta$ - 2 microglobulin values. Serum IL-6 concentrations were measured by using ELISA (Quantikine, R\&D Systems, USA). The detection limits for the assays were $0.8 \mathrm{mg} / \mathrm{dl}$ for CRP and $3.13 \mathrm{pg} / \mathrm{ml}$ for IL-6.

Statistical analysis. We used the Kolmogorov-Smirnov goodness of fit test to check the data for parametric distribution. Values obtained from patients were compared by the two- tailed Student's $t$-test or its non-parametric equivalent, the Mann-Whitney U test. For comparison of data from the same group of patients, but in different time intervals, we used the paired $t$-test. Values are expressed as median and range. A $p$ value of less than 0.05 was considered statistically significant.

\section{Results}

Clinical characteristics. Patients' characteristics are shown in Table 1 . The most striking difference was observed in lactic acid concentrations and this seems to be the result of sepsis-induced lactic acidosis. A total of 49 of 61 patients $(80.33 \%)$ had three or more signs of SIRS. Leukocyte count, pulse rate and respiratory rate were similar in both groups. High grade fever $\left(>38.5^{\circ} \mathrm{C}\right)$ on admission was detected mainly (19 of $22,86.4 \%$ ) in patients with bacterial infections, while 7 of the 27 non-septic patients $(25.9 \%)$ had a fever of $38.5^{\circ} \mathrm{C}$ or more.

Acute-phase proteins on admission. We detected an increase $(p<0.001)$ in baseline serum CRP concentrations in septic patients, although there were no overlapping values between the two groups. A smaller increase ( $p=0.0247)$ was noted with $\beta$-2 microglobulin but there was a considerable overlap in values between groups A and B. A threshold CRP value of $4 \mathrm{mg} / \mathrm{dl}$ could be used to define sepsis.

The highest significance $(p<0.0001)$ was detected in serum IL-6 levels, although there were no overlapping values between groups A and B (Table 2).

Patients with fever not due to sepsis did not differ significantly from the rest of the diabetics with no sepsis, concerning CRP and IL-6 values (CRP: median 2.7 , range $1.3-4.0 \mathrm{mg} / \mathrm{ml}$; IL-6: median 38.4 , range $20.4-61.6 \mathrm{pg} / \mathrm{ml}$ ).

Serum HPT, TRF and AAT, IgG, IgA, IgM and C3-C4 concentrations did not differ from normal values in all groups.

Acute-phase proteins during remission. Altogether 42 out of 49 patients $(85.7 \%)$ survived and were tested for acute-phase protein and IL-6 concentrations during remission. Five patients from group B died due to sepsis $(22.7 \%)$ and two patients from group A due to cardiopulmonary arrest $(7.4 \%)$. There was a decrease in serum CRP $(p=0.0034)$ and IL-6 $(p<0.0001)$ in group B compared with baseline values [Table 2].

CRP and IL-6 values as prognostic factors. Mortality was higher in septic diabetic patients (22.7 vs $7.4 \%$ ). Baseline CRP and IL-6 concentrations were higher in septic patients who died than in those who survived. C-Reactive protein in survivors ranged from 4.4 to $15.4 \mathrm{mg} / \mathrm{dl}$ (median 10.1) and in non-survivors from 15.0 to $21.9 \mathrm{mg} / \mathrm{dl}$ (median 21.9, $p<0.05$ ). Median IL-6 levels were $228.8 \mathrm{pg} / \mathrm{ml}$ in survivors (range $176.8-376.7 \mathrm{pg} / \mathrm{ml}$ ) and 467.2 in non-survivors (range 
Table 1. Patients' characteristics. Group A= diabetic patients with non-septic SIRS; Group B = diabetic patients with sepsis; $D K A$ diabetic ketoacidosis, $D H N K$ diabetic hyperosmolar non-ketotic coma (means \pm SEM)

\begin{tabular}{lccccc}
\hline Patients' characteristics & Group A (27 patients) & & \multicolumn{2}{c}{ Group B (22 patients) } \\
\cline { 2 - 3 } \cline { 5 - 6 } & DKA & DHNK & & DKA & DHNK \\
\hline Sex (male/female) & $8 / 7$ & $6 / 6$ & $5 / 7$ & $5 / 5$ \\
Mean age (years) & $47.3 \pm 12.2$ & $31.2 \pm 6.2$ & $52.2 \pm 9.8$ & $72.4 \pm 15.6$ \\
Glucose (mmol/l) & $16.6 \pm 2.4$ & $335.8 \pm 12.5$ & $17.5 \pm 2.1$ & $27.8 \pm 4.8$ \\
Osmolarity (moosm/l) & $296.2 \pm 20.8$ & $18.2 \pm 3.4$ & $293.2 \pm 16.8$ & $337.1 \pm 10.6$ \\
$\mathrm{HCO}_{3}(\mathrm{mEq} / \mathrm{l})$ & $10.4 \pm 3.1$ & $1.40 \pm 0.27 * \mathrm{a}$ & $8.3 \pm 1.9$ & $15.6 \pm 2.7$ \\
Lactic acid (mmol/l) & $1.32 \pm 0.24^{*}$ & $7.356 \pm 0.768$ & & $2.61 \pm 0.56^{* *}$ & $2.87 \pm 0.54^{* *}$ \\
pH & $7.187 \pm 0.345$ & & $7.126 \pm 0.720$ & $7.306 \pm 0.812$ \\
\hline
\end{tabular}

${ }^{*} p<0.05$

Table 2. Acute-phase proteins on admission and during remission. Group $\mathrm{A}=$ diabetic patients with non-septic SIRS; Group $\mathrm{B}=$ diabetic patients with sepsis; $D K A$ diabetic ketoacidosis, $D H N K$ diabetic hyperosmolar non-ketotic coma (median, range) Normal values: CRP: $<0.80 \mathrm{mg} / \mathrm{dL}, \beta-2$ microglobulin ( $\beta$-2 MG): 7-34 mg/dl; Haptoglobin (HPT): $50-220 \mathrm{mg} / \mathrm{dl}$; a-1 antithrypsin (ÁAT): 85-213 mg/dl; Transferrin (TRF): 252-429 mg/dl; Interleukin-6 (IL-6): 0-10.5 pg/ml

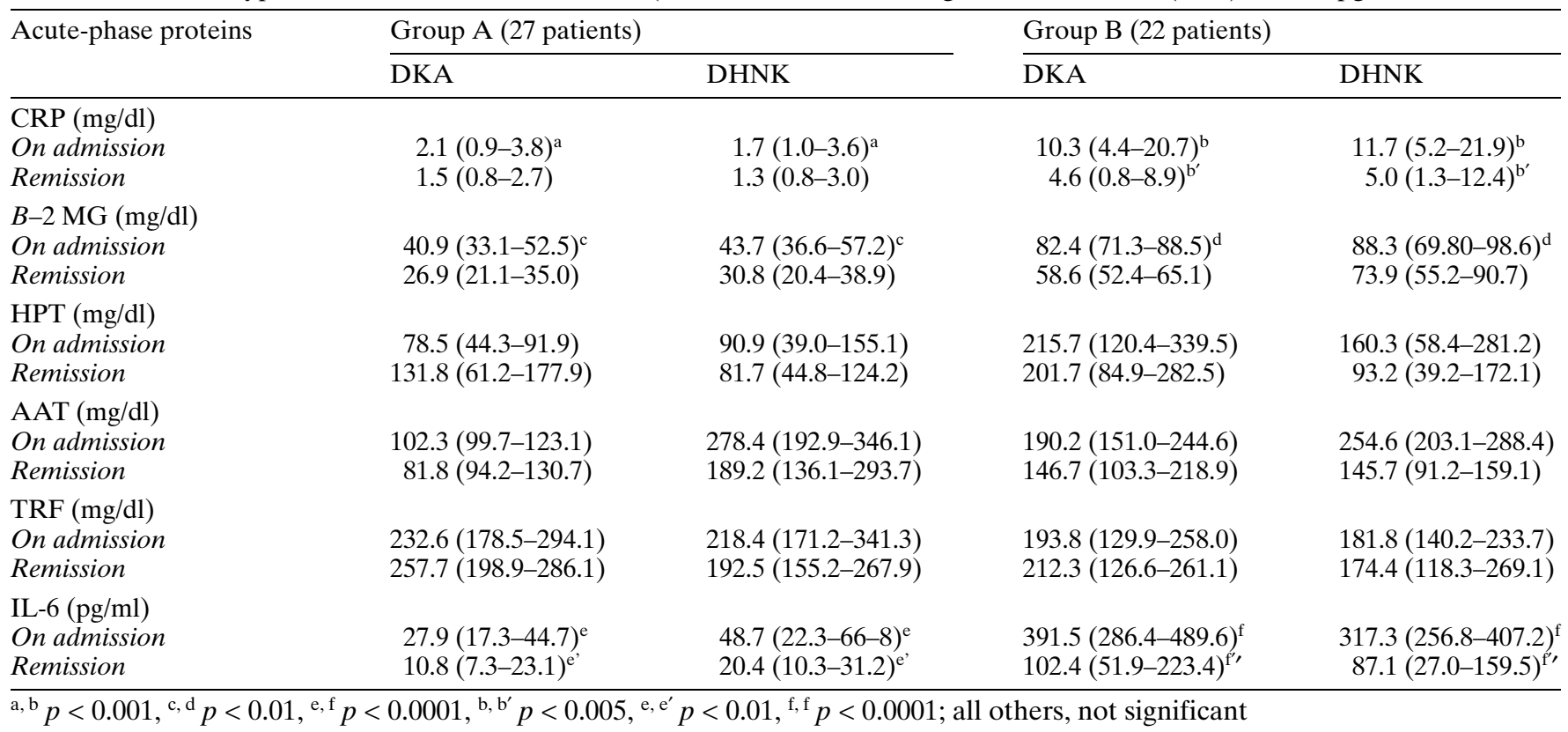

$328.8-489.6 \mathrm{pg} / \mathrm{ml}, p<0.01)$. In respect to other $\mathrm{pa}-$ rameters, baseline bicarbonate levels and arterial $\mathrm{pH}$ were lower $(p<0.05)$ in non-survivors, while age, sex, duration of diabetes and baseline osmolality and glucose concentrations were similar in both groups.

\section{Discussion}

Over-expressed acute inflammatory response as detected by a significant increase in serum CRP and IL6 seems to be a sensitive marker of sepsis in diabetic patients with SIRS due to ketoacidosis and hyperosmolar non-ketotic coma. Acute-phase proteins, mainly CRP, have been considered a valuable aid for the detection of sepsis in patients with diseases such as fever and bacteraemia [5], febrile children with cancer and neutropenia [6] and malaria infection [7]. High con- centrations of acute-phase proteins in septic patients were related to a parallel increase in IL-6, TNF and IL-1 b levels [8]. To our knowledge there is no information available as yet concerning the acute-phase reactants and IL-6 in DKA and DHNK stages.

Early detection of sepsis in patients with SIRS due to non-infectious causes (e.g. trauma, pancreatitis, neoplastic diseases etc) is sometimes difficult. Because infections are a frequent cause of death, especially if antibiotic treatment is delayed, many patients are over-treated with antibiotics resulting in side effects, induction of microbial resistance, super-infections and increases in costs and hospitalisation days. In this context, the use of a rapid, sensitive, specific and cheap test to detect sepsis early on could benefit these patients.

Diabetic ketoacidosis and hyperosmosis frequently lead in themselves to symptoms and signs reminis- 
cent of infection. On the other hand, it is known that infection precipitates diabetic ketoacidosis and coma. It is often difficult for the clinician to distinguish the septic patients from those with no infection. According to our data, 49 of the 61 patients $(80.33 \%)$ had SIRS but only 22 (36.06\%) had bacterial infection. Thus, clinical suspicion of infection could hardly be used, while culture-based confirmation of sepsis, when present, was available several hours or days after admission. In order to treat patients in the first stages of sepsis, we therefore tried to ascertain the diagnostic value of acute-phase proteins, especially CRP, together with IL-6.

We found that CRP was significantly higher in septic patients. As expected, an increase in CRP seems to be the result of the increase in IL- 6 production during infection, as septic patients had much higher serum IL-6 concentrations, with no overlapping values between septic and non-septic patients. We also observed that CRP together with IL-6 concentrations decreased significantly during remission, while b2 microglobulin remained increased. Both CRP and various cytokines, including IL-6, have previously been reported to predict the development of post-operative sepsis and multiple organ failure and as an indicator of septic complications and monitoring of sepsis $[9,10]$.

In conclusion, diabetic ketoacidosis and hyperosmosis can often be mistaken clinically for SIRS. Serial CRP and IL-6 measurements could be used to detect SIRS and treat sepsis in diabetic patients, in addition to other clinical tests and culture results. High CRP and IL-6 levels on admission seem to assist prognosis by indicating which patients need closer observation and ICU monitoring.

\section{References}

1. Gabay C, Kushner I (1999) Acute-phase proteins and other systemic responses to inflammation. N Engl J Med 340: 448-453

2. Claus DR, Osmand AP, Gewurz H (1976) Radioimmunoassay of human C-reactive protein and levels in normal sera. J Lab Clin Med 87: 120-128

3. Roine I, Banfi A, Bosch P, Ledermann W, Contreras C, Peltola H (1991) Serum C-reactive protein in childhood meningitis in countries with limited laboratory resources: a Chilean experience. Pediatr Infect Dis J 10: 923-928

4. Blackwell TS, Christman JW (1996) Sepsis and cytokines: current status. Br J Anaesth 77: 110-117

5. Diaz J, Arribas JM, Vallina E, Maradona SA, Hevia C, Blanko F (1992) Acute-phase reactants in sepsis. Rev Clin Esp 19: 473-477

6. Santolaya ME, Cofre J, Beresi V (1994) C-reactive protein: a valuable aid for the management of febrile children with cancer and neutropenia. Clin Infect Dis 18: 589-595

7. McGuire W, D'Alessandro U, Olaleye BO et al. (1996) Creactive protein and haptoglobin in the evaluation of a community based malaria control programme. Trans $\mathrm{R}$ Soc Trop Med Hyg 90: 10-14

8. Herrmann JL, Blanchard $\mathrm{H}$, Brunengo $\mathrm{P}$, Lagrange $\mathrm{PH}$ (1994) TNF-alpha, IL-1 b and IL-6 plasma levels in neutropenic patients after onset of fever and correlation with the C-reactive protein (CRP) kinetic values. Infection 22: 309-315

9. Yentis SM, Soni N, Sheldon J (1995) C-reactive protein as an indicator of resolution of sepsis in the intensive care unit. Intensive Care Med 21: 602-605

10. Gogos CA, Drosou E, Bassaris HP, Skoutelis A (2000) Proversus anti-inflammatory cytokine profile in patients with severe sepsis: a marker for prognosis and future therapeutic options. J Infect Dis 181: 180-184 